\title{
Thyroid: Physiology and Disease
}

\author{
Heiko Krude \\ Institute of Pediatric Endocrinology, Charité University Medicine Berlin, Berlin, Germany
}

\begin{abstract}
This year's search for thyroid publications has again revealed a number of more than 3,000 references under the search heading 'thyroid'. The selection for those papers which might have an impact for the pediatric endocrine community, either due to the general new information about thyroid function or due to its relevance for clinical purposes, ended up with a list of $0.8 \%$ of this large number of publications. Among these selections some 'streams of themes' of several papers under the same topic could be identified. The impact of selenium as an important trace element for the synthesis of deiodinases, and therefore for the regulation of the thyroid system, was one of these themes. The most relevant questions of pediatric thyroid hormone treatment were also touched this year with several papers focusing on the impact of thyroid hormone for prenatal, preterm and postnatal CNS development. The hottest topic in terms of impact was a 'burning paper' describing a new role for bile acids on energy expenditure, with the help of locally activated thyroid hormone in target cells.
\end{abstract}

\section{The hottest topic: bile burns fat and thyroid hormone is the heater}

\section{Bile acids induce energy expenditure by promoting intracellular thyroid hormone activation}

Watanabe M, Houten SM, Mataki C, Christoffolete MA, Kim BW, Sato H, Messaddeq N, Harney JW, Ezaki O, Kodama T, Schoonjans K, Bianco AC, Auwerx J

Institut de Génétique et Biologie Moléculaire et Cellulaire, CNRS/INSERM/ULP, Illkirch, France

Nature 2006;439:484-489

Background: While bile acids (BAs) have long been known to be essential in dietary lipid absorption and cholesterol catabolism, in recent years an important role for BAs as signaling molecules has emerged. BAs activate mitogen-activated protein kinase pathways, are ligands for the G-protein-coupled receptor (GPCR) TGR5 and activate nuclear hormone receptors.

Results: The authors show that the administration of BAs to mice increases energy expenditure in brown adipose tissue, preventing obesity and resistance to insulin. This novel metabolic effect of BAs is critically dependent on induction of the cyclic-AMP-dependent thyroid hormone activating enzyme type 2 iodothyronine deiodinase (D2) because it is lost in D2 ${ }^{-1-}$ mice. Treatment of brown adipocytes and human skeletal myocytes with BA increases D2 activity and oxygen consumption.

Conclusion: In both rodents and humans, the most important tissues for thermogenesis are specifically targeted by this mechanism because they coexpress D2 and TGR5. The BA-TGR5-cAMP-D2 signaling pathway is therefore a crucial mechanism for fine-tuning energy homeostasis that can be targeted to improve metabolic control.

Hyperthyroidism causes fat loss by activating metabolism. However, the systemic administration of thyroid hormones to overcome obesity is hampered by 'side effects' of hyperthyroidism on the heart, muscle, bone and apparently every other tissue. Moreover, hyperthyroid conditions enhance food intake at the same time so that fat loss is partly compensated. But what if we could induce tissuespecific generation of T3 rather than systemic hyperthyroidism? This paper shows that this may be possible: bile acids selectively increase thyroid hormone activity in fat-burning tissues by increasing the local deiodinase-2 expression, leading to increased local generation of the active thyroid hormone T3. Bile acid-based compounds as a treatment option for obesity would circumvent the side effects of systemic hyperthyroidism by the locally restricted activation of T3-induced metabolism. Envision yourself taking all you can eat while increasing the burning of calories with bile acids.

The term intracrinology was coined for the intracellular generation of estrogen from androgen in a target tissue that also expresses estrogen receptors. It was then used for the cortisone-cortisol shuttle 
in tissues with the glucocorticoid receptor. Yet, it all started with thyroidology. The following section will further focus on the newest knowledge about the capacity of the thyroid hormone system to regulate thyroid function by peripheral T4 to T3 conversion, which is the basis of this 'burning paper'.

\title{
Fine tuning of a sensitive system: seleno-deiodinases regulate thyroid hormone function
}

\begin{abstract}
This year has seen a number of publications focusing on the role of selenium and selenium cysteine proteins in thyroid physiology. Selenium plays a pivotal role for the enzymes deiodinase 1-3, which convert the precursor T4 into the active T3 or inactive reverse T3. Besides a 'selenium review of the century' with an impressive 669 references, an elegant knockout study in mice describes the consequences of selenium deprivation on thyroid function. Moreover, three high-impact papers described the roles of the three single selenium-dependent deiodinases. And finally the first 'disease' of selenium-related deiodinase deficiency was published, although it looks more like a laboratory change in thyroid hormone values rather than a real disease. A summary of this combined information is given in figure 1.
\end{abstract}

\section{Selenium, the thyroid, and the endocrine system}

Köhrle J, Jakob F, Contempre B, Dumont JE Institut für Experimentelle Endokrinologie, Charité, Humboldt Universität zu Berlin, Berlin, Germany josef.koehrle@charite.de

Endocr Rev 2005;26:944-984

Summary: Selenium (Se) can be part of proteins by building selenocysteine, which is incorporated into nascent proteins when a stop-codon UGA is followed by a particular 3'-hairpin-mRNA sequence. Recent identification of such selenocysteine-containing proteins has revealed relationships between the two trace elements Se and iodine and the hormone network. Several selenoproteins participate in the protection of thyrocytes from damage by $\mathrm{H}_{2} \mathrm{O}_{2}$ produced for thyroid hormone biosynthesis. Iodothyronine deiodinases are selenoproteins contributing to systemic or local thyroid hormone homeostasis. The Se content in endocrine tissues (thyroid, adrenals, pituitary, testes, ovary) is higher than in many other organs. Nutritional Se depletion results in retention, whereas Se repletion is followed by a rapid accumulation of Se in endocrine tissues, reproductive organs, and the brain. Furthermore, spermatogenesis depends on adequate Se supply, whereas Se excess may impair ovarian function. Comparative analysis of the genomes of several life forms reveals that higher mammals contain a limited number of identical genes encoding newly detected selenocysteine-containing proteins.

\section{Synthesis and metabolism of thyroid hormones is preferentially maintained in selenium-deficient transgenic mice}

Schomburg L, Riese C, Michaelis M, Griebert E, Klein MO, Sapin R, Schweizer U, Köhrle J

Institut für Experimentelle Endokrinologie, Charité-Universitätsmedizin Berlin, Berlin, Germany

lutz.schomburg@charite.de

Endocrinology 2006;147:1306-1313

Background: The thyroid gland is rich in selenium (Se) and expresses a variety of selenoproteins that are involved in antioxidative defense and metabolism of thyroid hormones (TH). Se deficiency impairs regular synthesis of selenoproteins and adequate TH metabolism. Recently, a mouse model was generated that lacks the plasma Se carrier, selenoprotein P (SePP). SePP-knockout mice display decreased serum Se levels and manifest growth defects and neurological abnormalities partly reminiscent of thyroid gland dysfunction or profound hypothyroidism. In the present paper the TH axis in developing and adult SePP-knockout mice was described. 


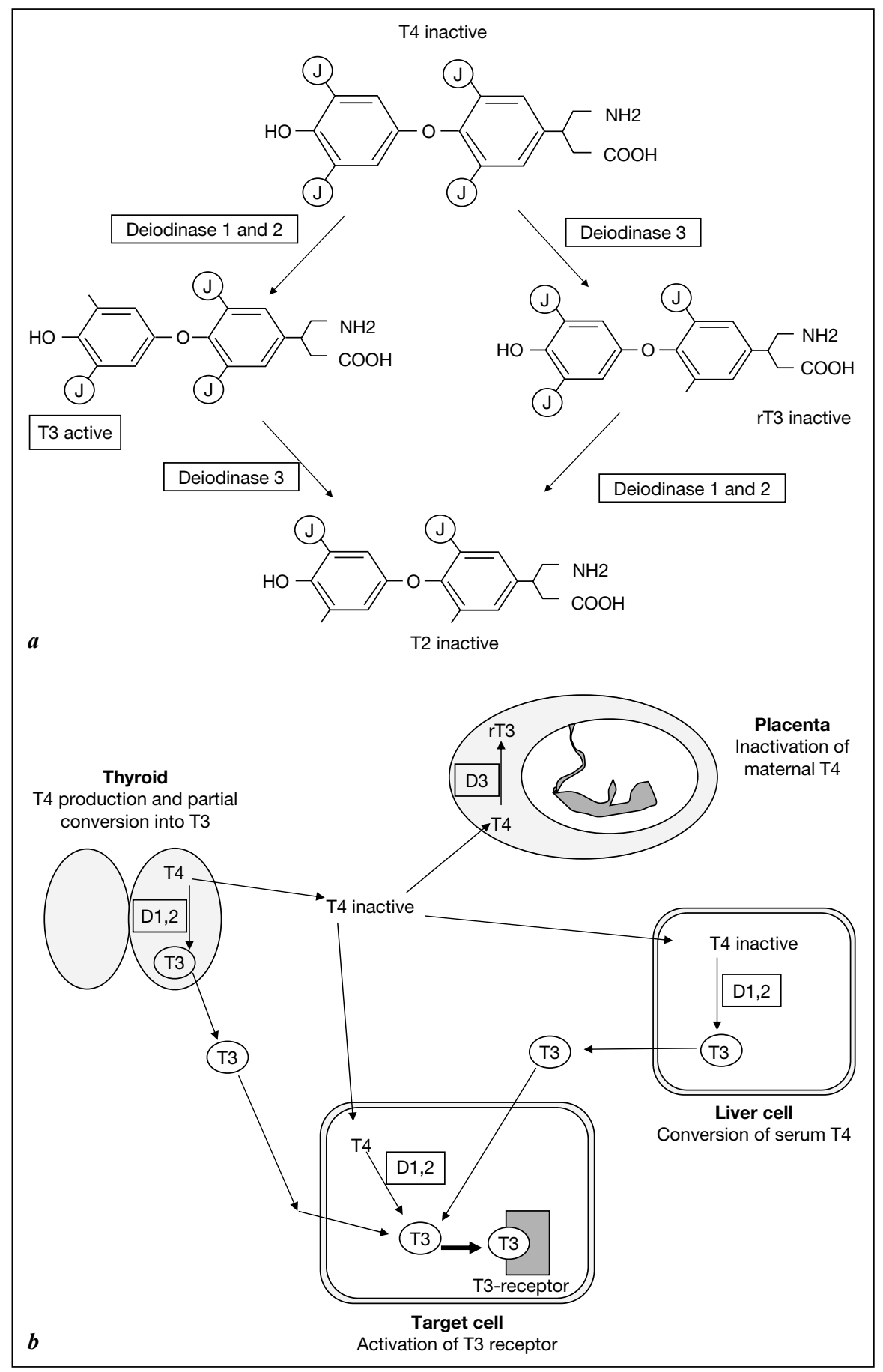

Fig. 1. $a$ Production and conversion of T4. $b$ Production and conversion of T4 in different tissues.

Results: Surprisingly, expression of Se-dependent $5^{\prime}$-deiodinase type 1 was only slightly altered in liver, kidney, or thyroid at postnatal day 60 , and $5^{\prime}$-deiodinase type 2 activity in brain was normal in SePP-knockout mice. Thyroid gland morphology, thyroid glutathione peroxidase activity, thyroid Se concentration, and serum levels of TSH, T4, or T3 were within a normal range. Pituitary TSH- $\beta$ 
transcripts and hepatic 5'-deiodinase type $1 \mathrm{mRNA}$ levels were unchanged, indicating regular T3 bioactivity in thyrotropes and hepatocytes. Cerebellar granule cell migration as a sensitive indicator of local T3 action during development was undisturbed.

Conclusion: Collectively, these findings demonstrate that low levels of serum Se or SePP in the absence of other challenges do not necessarily interfere with regular functioning of the TH axis. 5'-Deiodinase isozymes are preferentially supplied, and Se-dependent enzymes in the thyroid are even less dependent on serum levels of Se or SePP than in brain. This indicates that the thyroid gland and its selenoenzymes are highly prioritized with respect to Se supply within the organism.

This paper is a nice example of how unexpected results of a complex experiment can still be informative. Despite selenium deprivation the authors found that the thyroid phenotype is normal. However, this does not exclude a crucial role of selenium for thyroid function and the authors show that nature has installed an emergency system which still supplies thyroid with the last molecules of selenium available to keep the thyroid hormone production going. It seems that in states of severe selenium deficiency the thyroid is still provided with a mechanism to extract as much selenium as possible to proceed with normal thyroid hormone function.

\section{Targeted disruption of the type 1 selenodeiodinase gene (Dio1) results in marked changes in thyroid hormone economy in mice}

Schneider MJ, Fiering SN, Thai B, Wu SY, St Germain E, Parlow AF, St Germain DL, Galton VA

Department of Physiology, Dartmouth Medical School, Lebanon, N.H., USA

Endocrinology 2006;147:580-589

Background: Type 1 deiodinase (D1) is thought to be an important source of T3 in the euthyroid state. To explore the role of the D1 in thyroid hormone economy, a D1-deficient mouse (D1KO) was made by targeted disruption of the Dio1 gene.

Results: The general health and reproductive capacity of the D1KO mouse were seemingly unimpaired. In serum, levels of T4 and rT3 were elevated, whereas those of TSH and T3 were unchanged, as were several indices of peripheral thyroid status. It thus appears that the D1 is not essential for the maintenance of a normal serum T3 level.

Conclusion: The authors conclude that although the D1 is of questionable importance to the well-being of euthyroid mice, it may play a major role in limiting the detrimental effects of conditions that alter normal thyroid function, including hyperthyroidism and iodine deficiency.

\section{Type 2 iodothyronine deiodinase is the major source of plasma T3 in euthyroid humans}

Maia AL, Kim BW, Huang SA, Harney JW, Larsen PR

Endocrine Division, Hospital de Clinicas de Porto Alegre, Universidade Federal do Rio Grande do Sul, Porto Alegre,

Brazil

J Clin Invest 2005;115:2524-2533

Background: As shown in the previous paper, it seems unclear what the relative roles of types 1 and 2 iodothyronine deiodinases (D1 and D2) in extrathyroidal T3 production are. Therefore, the authors calculated the rate of thyroxine (T4) to T3 conversion by intact cells transiently expressing D1 or D2 at low $(2 \mathrm{p} M)$, normal $(20 \mathrm{pM})$, and high $(200 \mathrm{p} M)$ free T4 concentrations.

Results: The authors predict that in euthyroid humans, D2-generated T3 is $29 \mathrm{nmol} /$ day, while D1-generated T3 is $15 \mathrm{nmol} /$ day. The total estimated extrathyroidal T3 production from major deiodinase-expressing tissues, $44 \mathrm{nmol} / \mathrm{day}$, is in close agreement with the $40 \mathrm{nmol} \mathrm{T3} /$ day based on previous kinetic studies. D2-generated T3 production accounts for approximately $71 \%$ of the peripheral T3 production in hypothyroidism, but D1 for approximately $67 \%$ in thyrotoxic patients.

Conclusion: The authors conclude that the intracellular D2-generated T3 has a greater effect on T3dependent gene transcription than that from D1, which indicates that generation of nuclear T3 is an intrinsic property of the D2 protein. They suggest that impairment of D2-generated T3 is the major cause of the reduced $\mathrm{T} 3$ production in the euthyroid sick syndrome. 


\section{Type 3 deiodinase is critical for the maturation and function of the thyroid axis}

Hernandez A, Martinez ME, Fiering S, Galton VA, St Germain D

Department of Medicine, Dartmouth Medical School, Lebanon, N.H., USA

Arturo.Hernandez@Dartmouth.edu

J Clin Invest 2006;116:476-484

Background: Developmental exposure to appropriate levels of thyroid hormones in a timely manner is critical to normal development in vertebrates. Deiodinase type 3 (D3) degrades T4 into reverse T3 and T3 into T2. and is highly expressed in the pregnant uterus, placenta, and fetal and neonatal tissues. Thus, D3 is among the factors potentially protecting fetal tissues against high thyroid hormone levels since maternal T4 levels are higher than fetal levels (e.g. in rats 20 times higher). To determine the physiological role of D3, the authors have generated a mouse D3 knockout model (D3KO) by a targeted inactivating mutation of the Dio3 gene in mouse ES cells.

Results: Early in life, D3KO mice exhibit delayed 3,5,3'-triiodothyronine (T3) clearance, a markedly elevated serum T3 level, and overexpression of T3-inducible genes in the brain. From postnatal day 15 to adulthood, D3KO mice demonstrate central hypothyroidism, with low serum levels of 3,5,3', 5 '-tetraiodothyronine (T4) and T3, and modest or no increase in thyroid-stimulating hormone (TSH) concentration. Peripheral tissues are also hypothyroid. Hypothalamic T3 content is decreased while thyrotropin-releasing hormone (TRH) expression is elevated.

Conclusion: These results demonstrate that the lack of D3 function results in neonatal thyrotoxicosis followed later by central hypothyroidism that persists throughout life. These mice provide a new model of central hypothyroidism and reveal a critical role for D3 in the maturation and function of the thyroid axis.

The first two papers confirm that the two activating deiodinases, which convert T4 into active T3, are redundant as they intermingle in their function, and that most likely both enzymes can more or less compensate the loss for each other. Many attempts have failed so far to inactivate the forward strand coding for deiodinase-3 gene, while keeping the reverse strand intact that codes for the antisense transcript Dio3 with an unknown function. However, this has now been overcome and the third paper defines the role of the third deiodinase: it is mainly responsible for protecting the growing fetus against high maternal T4 levels. In unprotected D3-deficient mice, fetal hyperthyroidism due to elevated T3 suppresses TSH synthesis. Whereas neonatal hyperthyroidism lasts for several days postnatally, it results in permanent central hypothyroidism. This condition is comparable to the long-lasting central hypothyroid state that was recently described for infants born to mothers with uncontrolled hyperthyroidism due to Graves' disease. It will not be long before we hear of human cases of D3 deficiency; the race is open to all. Growth and neuronal gene expression is markedly changed in this new 'fetal hyperthyroidism model' of D3-deficient mice, and it will be interesting to determine if the developmental outcomes of equivalent children will be reduced.

\section{Mutations in SECISBP2 result in abnormal thyroid hormone metabolism}

Dumitrescu AM, Liao XH, Abdullah MS, Lado-Abeal J, Majed FA, Moeller LC, Boran G, Schomburg L, Weiss RE, Refetoff $S$

Department of Human Genetics, University of Chicago, Chicago, III., USA

Nat Genet 2005:37:1247-1252

Background: Until now no genetic defect in patients could be identified which affect the selenocysteinedeiodinase system. The authors were confronted with healthy 'patients' who are characterized by a so far unknown combination of circulating thyroid hormone abnormalities consisting in increased T4, fT4, and reverse T3 but low T3, while TSH was slightly elevated. Due to this profile, they speculated on a defect in deiodinase 1 or 2 activity leading to reduced conversion of T4 into T3, while inactivation of T4 into reverse T3 is maintained.

Results: Fibroblasts of the patients showed decreased DIO2 enzymatic activity but genetic testing could not link the phenotype to a defect in the deiodinase locus. Thereafter a systematic linkage analysis of genes involved in DIO2 synthesis and degradation led to the identification of an inherited Sec incorporation defect, caused by a homozygous missense mutation in SECISBP2 (also called SBP2). Because SBP2 is a crucial cofactor for selenoprotein synthesis, these defects had a generalized effect on selenoproteins. 
Conclusion: Despite the generalized effect of the mutant cofactor for selenoprotein synthesis, the authors concluded that the mild phenotype of the patients (transient growth retardation and mild thyroid hormone changes) is due to an incomplete loss of SBP2 function.

In eukaryotes, decoding of the UGA codon as selenocysteine (sec) requires a sec insertion sequence (SECIS) in the 3'-untranslated region of the mRNA. The nickname for the impossible name SECISBP2 is SBP2. The matter of deiodinases and thyroid hormone activation by selenoenzymes is still puzzling. Besides the new defect in SBP2, which leads to a mild alteration of thyroid hormone concentrations without an obvious clinical phenotype, no genetic disease has so far be attributed to a defect in selenoprotein enzymes. This does not implicate a less important role but rather a redundancy of an important system of an intracrine activation and regulation of thyroid hormone function by deiodinases.

\title{
The impact of thyroid hormone on brain development: the more the better?
}

\begin{abstract}
It is remarkable how neuronal function is critically dependent on the thyroid hormone. It is only recently that the role of thyroid hormone during embryogenesis has come into focus, and it seems that the maternal thyroid hormone supply is critical for the normal CNS development of human embryos. According to the paper by Kooistra et al. (see below), a mild maternal hypothyroxinemia in the first trimester may delay neonatal developmental milestones. As much as it is protected by the placenta from hyperthyroxinemia, the human embryo needs a maternal (= external) thyroid hormone supply for its early steps of development before its own thyroid axis becomes active. Later during fetal life a less vulnerable phase begins, and it becomes increasingly evident that children with congenital (and fetal) hypothyroidism will develop normally when postnatal thyroid hormone substitution is optimized (see papers by Bongers-Schokking and Selva). Intriguingly, Bongers-Schokking showed that the more thyroid hormone substituted the better the outcome in terms of IQ points, even when TSH is suppressed. In contrast to these optimistic findings, the extrauterine fetal life of a preterm newborn creates such an unbalanced situation of thyroid hormone supply that the question of whether more or less thyroid hormone supply in this condition would be beneficial or not will need more extensive clinical research (see Williams and van Wassenaer).
\end{abstract}

\section{Neonatal effects of maternal hypothyroxinemia during early pregnancy}

Kooistra L, Crawford S, van Baar AL, Brouwers EP, Pop VJ

Department of Pediatrics, University of Calgary, Calgary, Alta., Canada

libbe.kooistra@calgaryhealthregion.ca

Pediatrics 2006;117:161-167

Background: To describe the effect of suboptimal maternal thyroid hormone supply to a growing embryo the authors sought to examine the neurobehavioral profile of neonates who are born to women with hypothyroxinemia during early pregnancy. They examined 108 neonates who were born to mothers with low maternal free thyroid hormone (fT4 concentrations; <10th percentile) at 12 weeks' gestation (case patients) and 96 neonates who were born to women whose fT4 values were between the 50th and 90th percentiles, matched for parity and gravidity (control subjects). Newborn development was assessed at 3 weeks of age using the Neonatal Behavioral Assessment Scale. Maternal thyroid function (fT4 and thyrotropin hormone) was assessed at 12, 24, and 32 weeks' gestation.

Results: Infants of women with hypothyroxinemia at 12 weeks' gestation had significantly lower scores on the Neonatal Behavioral Assessment Scale orientation index compared with subjects. Regression analysis showed that first-trimester maternal fT4 but not maternal TSH or fT4 later in gestation was a significant predictor of orientation scores.

Conclusion: This study confirms that maternal hypothyroxinemia constitutes a serious risk factor for neurodevelopmental difficulties that can be identified in neonates as young as 3 weeks of age. 
Thyroid hormone has a fundamental impact on neuronal development. Because early embryonal development of the CNS takes place in a period without a functional thyroid gland, thyroid hormone needs to be transferred from the maternal circulation. Therefore, a maternal deficit of available thyroid hormone might substantially interfere with normal CNS development. The finding of this paper, showing that very early developmental parameters are significantly poorer in 3-week-old neonates with a history of low maternal fT4 in the first trimester, suggests that very early steps of CNS development are vulnerable to fetal hypothyroidism, and cannot be compensated later during fetal life by sufficient thyroid hormone production by the fetus. The practical implications are that close monitoring of thyroid function throughout pregnancy and careful adjustment of the thyroid supplementation dosage are warranted for hypothyroid pregnant women in order to avoid the potential ill effects of an insufficient availability of thyroid hormone to the fetus.

\title{
Serum thyroid hormones in preterm infants: associations with postnatal illnesses and drug usage
}

\author{
Williams FL, Ogston SA, van Toor H, Visser TJ, Hume R \\ Community Health Sciences, University of Dundee, Ninewells Hospital and Medical School, Dundee, UK \\ J Clin Endocrinol Metab 2005;90:5954-5963
}

Background: Transient hypothyroxinemia is common in infants $<30$ weeks' gestation and is associated with neurodevelopmental deficits. Reductions in T4 and T3 levels with TSH unchanged are the key features of severe illness. The authors intended to investigate the contribution of postnatal factors to the variations in serum levels of iodothyronines, thyroid-binding globulin, and TSH. They recruited a cohort of 780 infants (23-34 weeks' gestation) involving eleven level III Scottish neonatal intensive care units. Results: Serum levels of TSH, free T4, T3, and T4 are variably but significantly associated with bacteremia, endotracheal bacterial cultures, persistent ductus arteriosus, necrotizing enterocolitis, cerebral ultrasonography changes, oxygen dependence at 28 days, and the use of aminophylline, caffeine, dexamethasone, diamorphine, and dopamine.

Conclusion: There are many more associations of postnatal factors with transient hypothyroxinemia than had previously been considered in preterm infants including those drugs that interfere with the hypothalamic-pituitary-thyroid axis, e.g. dopamine.

It seems difficult to consider which thyroid hormone level would be optimal for postnatal development in preterm babies, while outside the protective environment of the uterus. But now this study tells us that it may be impossible to ever answer this question. The postnatal factors that change the levels of thyroid hormones include the entire pathology of prematurity. All of the following variables are to be considered when analyzing thyroid hormones: bacteremia, endotracheal bacterial cultures, persistent ductus arteriosus, necrotizing enterocolitis, cerebral ultrasonography changes, oxygen dependence at 28 days, and the use of aminophylline, caffeine, dexamethasone, diamorphine, and dopamine. The current study investigated a cohort of 780 preterm newborns. At least this size of a cohort might be required to perform a treatment trial with the power to solve this question.

\section{Ten-year follow-up of children born at $<\mathbf{3 0}$ weeks' gestational age supplemented with thyroxine in the neonatal period in a randomized, controlled trial}

van Wassenaer AG, Westera J, Houtzager BA, Kok JH

Department of Neonatology, Emma Children's Hospital Academic Medical Center, Amsterdam, The Netherlands a.vanwassenaer@amc.uva.nl

Pediatrics 2005; 116:613-618

Background: Thyroid hormones are essential for brain development. The authors conducted a randomized, controlled trial with thyroxine (T4) supplementation in infants $<30$ weeks' gestation and with the last neurodevelopmental follow-up moment at the age of 5.5 years. T4 supplementation was associated with improved outcome of infants $<28$ weeks' gestation and worse outcome of infants of 29 weeks' gestation. Gestational age-dependent effects of T4 supplementation at the mean age of 10.5 years were evaluated in children participating in a randomized, controlled trial. 
Methods: Questionnaires regarding school outcome, behavior, quality of life, motor problems, and parental stress were sent to the parents and children and their teachers at the same time point for all surviving children (9-12 years of age).

Results: $72 \%$ of the families responded to the questionnaires. Non-respondents had more sociodemographic risk factors and worse development until 5.5 years. At the mean age of 10.5 years, T4 supplementation was associated with better school outcome in those who were $<27$ weeks' gestation and better motor outcome in those who were $<28$ weeks' gestation, whereas the reverse was true for those who were born at 29 weeks' gestation. No other gestational age-dependent outcomes were found.

Conclusion: Gestation-dependent effects of T4 supplementation remain stable over time. These do not prove beneficial effects of T4 in infants $<28$ weeks but should be the background for a new randomized, controlled trial with thyroid hormone in this age group.

Five years ago the same authors provided a study indicating that in preterm neonates $T 4$ supplementation was associated with improved outcome of infants $<28$ weeks' gestation and worse outcome of infants of 29 weeks' gestation. Now it is impressive that even after 10 years the significant differences in developmental timing in the two treatment groups remain stable. This initial trial is a real milestone because it shows the burden and benefits of a treatment with thyroid hormone in preterm children, and we have to wait until the new study will give us better arguments for routine thyroid hormone treatment in preterm newborns.

\section{Influence of timing and dose of thyroid hormone replacement on mental, psychomotor, and behavioral development in children with congenital hypothyroidism}

Bongers-Schokking JJ, de Muinck Keizer-Schrama SM

Department of Endocrinology, Erasmus Medical Center, Sophia Children's Hospital, Rotterdam, The Netherlands abongers@worldonline.nl

J Pediatr 2005;147:768-774

Background: To evaluate the influence of initial and postinitial treatment factors on cognitive, psychomotor, and psychological outcome in schoolchildren with congenital hypothyroidism $(\mathrm{CH})$.

Methods: The authors studied 45 patients (19 with severe $\mathrm{CH}$ and 26 with mild $\mathrm{CH}$ ) and 37 control children by correlating initial and postinitial treatment factors (free thyroxine and thyroid-stimulating hormone [TSH] concentrations, and the percentage of overtreatment and undertreatment periods) with the results of neuropsychological tests and behavior (as reported on the Teacher Report Form [TRF]).

Results: The global IQ of the children with $\mathrm{CH}$ was comparable to that of the controls; visuomotor and verbal scores were lower, and total TRF scores were higher. Ethnic group, previous development, and overtreatment predicted IQ and verbal scores, with higher scores seen for the overtreated patients than for the control children and those patients who had not been overtreated. As initial treatment was less satisfactory, total TRF scores were higher.

Conclusion: The study suggests that initial and postinitial suboptimal treatment of $\mathrm{CH}$ leads to abnormalities in IQ and specific fields. Overtreatment may advance cognitive development in $5 \frac{1}{2} 2^{-}$to 7 -year-olds. Suboptimal initial treatment may lead to behavioral problems.

\section{Neurodevelopmental outcomes in congenital hypothyroidism: comparison of initial T4 dose and time to reach target T4 and TSH}

Selva KA, Harper A, Downs A, Blasco PA, Lafranchi SH

Children's Diabetes \& Endocrine Center, Legacy Emanuel Hospital, Portland, Oreg., USA

kselva@lhs.org

J Pediatr 2005;147:775-780

Background: To ultimately answer the question which thyroid hormone dose is the best for $\mathrm{CH}$ neonates. Methods: The authors compared neurodevelopmental outcomes in severe and moderate congenital hypothyroidism $(\mathrm{CH})$ among three different initial $L$-thyroxine doses and examined the effect of the time to thyroid function normalization on neurodevelopmental outcomes in a prospective study in 31 subjects including the Mullen Scales of Early Learning, Wechsler Preschool and Primary Scale of 
Intelligence-Revised, Wechsler Intelligence Scale for Children, Wide-Range Achievement Test, and Child Behavioral Checklist.

Results: Subjects started on higher initial $L$-thyroxine doses $(50 \mu \mathrm{g})$ had full-scale IQ scores 11 points higher than those started on lower $(37.5 \mu \mathrm{g})$ initial doses. However, verbal IQ, performance IQ, and achievement scores did not differ among the three treatment cohorts. Subjects with moderate $\mathrm{CH}$ had higher full-scale IQ scores than subjects with severe $\mathrm{CH}$, regardless of the initial treatment dose. Subjects who took longer than 2 weeks to normalize thyroid function had significantly lower cognitive, attention, and achievement scores than those who achieved normal thyroid function at 1 or 2 weeks of therapy.

Conclusion: Initial $L$-thyroxine dose and faster time to normalization of thyroid function are important to optimal neurodevelopmental outcome. In severe $\mathrm{CH}$, it is important to choose an initial dose at the higher end of the recommended range to achieve these goals.

For years we were uncertain whether we should normalize T4 (or T3) levels or rather suppress TSH. It seems that the latter is more favorable in terms of neurocognitive development. These two cohorts are telling us that the second generation of $\mathrm{CH}$ children treated now with $50 \mathrm{mg}$ L-thyroxine/day are doing better than the first generation of $\mathrm{CH}$ children who were treated with $25-37 \mathrm{mg}$ and who still had some IQ point deficits, even if in the normal range. Very nicely the Dutch group has now excluded the concern that higher thyroid doses might result in a less favorable outcome. They show in their study that those children with the highest dose and the most frequently suppressed TSH levels have the best IQ values of the whole cohort. This does not mean that the more the better, since we know that hyperthyroidism in neonatal life also has a negative impact on brain development. But moderate overdoses of $L$-thyroxine which suppress TSH are obviously not harmful and the reflex to reduce the dose in these infants with suppressed TSH instead of continuing with the higher 50-mg dose might be the more harmful decision.

\title{
The effect of thyroxine treatment started in the neonatal period on development and growth of two-year-old Down syndrome children: a randomized clinical trial
}

\author{
van Trotsenburg AS, Vulsma T, van Rozenburg-Marres SL, van Baar AL, Ridder JC, Heymans HS, \\ Tijssen JG, de Vijlder JJ \\ Academic Medical Center, University of Amsterdam, Department of Pediatric Endocrinology, Amsterdam, \\ The Netherlands \\ a.s.vantrotsenburg@amc.uva.nl \\ J Clin Endocrinol Metab 2005;90:3304-3311
}

Background: Young Down syndrome children appear to have a mild form of congenital hypothyroidism that is rarely detected by neonatal screening and usually left untreated.

Methods: The authors intended to investigate the effects of thyroxine treatment on development and growth of young Down syndrome children in a single-center, randomized, double-blind, 24-month trial in 196 Down syndrome neonates. Neonates were randomly assigned to treatment for 2 years with either thyroxine $(\mathrm{n}=99$; initial dose $8 \mu \mathrm{g} / \mathrm{kg})$ or placebo $(\mathrm{n}=97)$. The primary outcome was mental and motor development at age 24 months, assessed with the Bayley Scales of Infant Development II. Results: At age 24 months, the developmental testing results of 90 thyroxine- and 91 placebo-treated children were available for analysis. The thyroxine-treated children had a 0.7 -month smaller delay in motor developmental age (95\% confidence interval [CI] -1.4 to 0$)$, corresponding to a difference of seven motor developmental index points. The mental developmental age delay was also 0.7 month smaller in the thyroxine group $(95 \%$ CI -1.5 to 0.2$)$, but lacked statistical significance. Thyroxinetreated children had greater gains in length $(1.1 \mathrm{~cm} ; 95 \%$ CI $0.2-2.0)$ and weight $(378 \mathrm{~g} ; 95 \%$ CI 55-701).

Conclusion: The data of this study provide evidence that thyroxine treatment may improve development and growth of young Down syndrome children.

Mental development in Down syndrome children is not retarded due to severe hypothyroidism, but rather to overexpression in the brain of genes of the third 21st chromosome. The mild and often fluctuating increases of TSH in Down syndrome children might mirror some regulatory disturbances of 
the thyroid axis and are not the sign of overt hypothyroidism. However, a non-optimal thyroid function might in part contribute to some aspects of developmental delay. This paper has now calculated this effect in a randomized study, which shows that optimization of thyroid hormone levels in Down syndrome children improves their development by 0.7 month at age 2 years, which was mildly significant for motor development and insignificant for mental development. Is this of any importance in the frame of an overall delay at the age of 2 years in these Down syndrome children of 12 months? Does it justify the treatment with LT4? These results can be discussed and a decision taken with the parents.

\title{
Nothing new: congenital hypothyroidism unsolved, but a new concern
}

\section{Extending the clinical heterogeneity of iodide transport defect (ITD): a novel mutation $\mathbf{r 1 2 4 h}$ of the sodium/iodide symporter gene and review of genotype-phenotype correlations in ITD}

\author{
Szinnai G, Kosugi S, Derrien C, Lucidarme N, David V, Czernichow P, Polak M \\ Service d'Endocrinologie Pédiatrique and Institut National de la Sante et de la Recherche Médicale, Equipe Mixte \\ INSERM 0363, Hôpital Necker Enfants Malades, Paris, France \\ michel.polak@nck.ap-hop-paris.fr
}

J Clin Endocrinol Metab 2006;91:1199-1204

Background: ITD is an autosomal recessive disorder resulting in varying degrees of congenital hypothyroidism $(\mathrm{CH})$ with goiter, low or absent radioiodide uptake (RIUT) as determined by thyroid scintigraphy, and low iodide saliva-to-plasma ratio. Defects of the sodium/iodide symporter gene (NIS) have been shown to cause ITD. The molecular studies of NIS in a patient with ITD and genotype-phenotype correlation analysis in 31 patients with NIS defects reported worldwide are described.

Results: In a newborn with symptomatic $\mathrm{CH}$ and a large goiter, thyroid scintigraphy showed no RIUT $(0 \%)$. NIS sequencing identified the new homozygous mutation $\mathrm{R} 124 \mathrm{H}$ in exon 2 . This mutation was associated with abolition of iodide uptake in vitro when transfected in COS-7 cells. Immunocytochemical studies documented correct targeting of the mutated protein to the plasma membrane of transfected cells. Genotype-phenotype correlation analysis showed that the onset of hypothyroidism occurred in the neonatal period with four NIS mutations (neonatal-onset genotypes), during infancy with 3 NIS mutations (infancy-onset genotypes), and during childhood with 3 NIS mutations (childhood-onset genotypes). RIUT is a direct measure of residual NIS activity in vivo. Mean RIUT was lower in patients with the neonatal-onset genotypes $(0.88 \pm 0.2 \%)$ than the infancy-onset genotypes $(1.9 \pm 0.4 \%$, $\mathrm{p}<0.05)$ and childhood-onset genotypes $(2.6 \pm 0.7 \%, \mathrm{p}<0.05)$.

Conclusions: Genotype-phenotype correlations suggest that age at hypothyroidism onset may be genotype-specific and may depend on genotype-specific residual NIS activity.

\section{Missense mutation in the transcription factor NKX2-5: a novel molecular event in the pathogenesis of thyroid dysgenesis}

Dentice M, Cordeddu V, Rosica A, Ferrara AM, Santarpia L, Salvatore D, Chiovato L, Perri A, Moschini L, Fazzini C, Olivieri A, Costa P, Stoppioni V, Baserga M, De Felice M, Sorcini M, Fenzi G, Di Lauro R, Tartaglia M, Macchia PE Dipartimento di Endocrinologia ed Oncologia Molecolare e Clinica, Università di Napoli Federico II, Naples, Italy pmacchia@unina.it

J Clin Endocrinol Metab 2006;91:1428-1433

Background: Congenital hypothyroidism $(\mathrm{CH})$ is a common endocrine disorder with an incidence of 1:3-4,000 at birth. In $80-85 \%$ of cases, $\mathrm{CH}$ is caused by defects in thyroid organogenesis, resulting in absent, ectopically located and/or severely reduced gland (thyroid dysgenesis, TD). Mutations in genes controlling thyroid development have demonstrated that, in a few cases, TD is a mendelian trait. However, accumulating evidence supports the view that the genetics of TD is complex, possibly with a polygenic/multifactorial basis. A higher prevalence of congenital heart disease (CHD) has been documented in children with $\mathrm{CH}$ than in the general population. Such an association suggests a possible 
pathogenic role of genes involved in both heart and thyroid development. NKX2-5 encodes a homeodomain-containing transcription factor with a major role in heart development, and mutations affecting this gene have been reported in individuals with CHD.

Results: The authors show that $\mathrm{Nkx} 25^{(-1-)}$ embryos exhibit thyroid bud hypoplasia, providing evidence that NKX2-5 plays a role in thyroid organogenesis, and that NKX2-5 mutations contribute to TD. NKX2-5 mutational screening in 241 patients with TD allowed the identification of three heterozygous missense changes (R25C, A119S and R161P) in 4 patients with TD. Functional characterization of the three mutations demonstrated reduced DNA binding and/or transactivation properties with a dominant negative effect on wild-type NKX2-5.

Conclusion: These data suggest a previously unknown role of NKX2-5 in the pathogenesis of TD.

\begin{abstract}
Although $\mathrm{CH}$ is the most prevalent congenital endocrine defect, we are still devoid of a mechanism in most cases. A few genetic defects were described in the last 10 years. Most prominent are the recessively inherited biosynthesis defects (dyshormonogenesis), which explain $10-15 \%$ of all cases. Beside mutations in the thyroid peroxidase and thyroglobulin genes, defects of the sodium iodine symporter NIS were among this group of classical genetic defects. This year we learnt that NIS mutations can be non-apparent at birth and result in goiters and hypothyroidism later in life, presenting as iodine deficiency. This finding provokes the question of whether goiters in mild iodine-deficient regions might indicate mild NIS defects?

Within the group of developmental genes, which can cause thyroid dysgenesis, we welcome a new member: Paolo Macchia's group found the first mutations in the transcription factor NKX2.5 that plays a role in thyroid as well as heart development. We know now of five genes that may cause thyroid dysgenesis: PAX8, NKX2.1/TITF1, FOXE1/TTF2, TSHR, NKX2.5. Nevertheless, the number of solved cases in this larger group of $85 \%$ patients with $\mathrm{CH}$ and thyroid dysgenesis is very low. Only a few individual patients could be identified with mutations in these five candidate genes. So we wait with tension for the cause of the remaining ones and until then think about other non-genetic causes.
\end{abstract}

\title{
High incidence of thyroid cancer in long-standing goiters with thyroglobulin mutations
}

Hishinuma A, Fukata S, Kakudo K, Murata Y, leiri T

Department of Clinical Laboratory Medicine, Dokkyo University School of Medicine, Mibu, Tochigi, Japan

a-hishi@dokkyomed.ac.jp

Thyroid 2005;15:1079-1084

Background: Mutations of the thyroglobulin gene cause defective thyroid hormone synthesis, resulting in congenital hypothyroidism. Since the first report in 1999 on the thyroglobulin mutations C1264R and C1996S, 14 adult patients ( 7 males and 7 females) from 9 unrelated families were identified by this group. All patients had huge goiters that first appeared in childhood. Persistent growth of the thyroid gland resulted in multiple operations.

Results: Of 11 patients who had undergone surgery, 7 had thyroid cancers. Of 5 patients whose thyroid tissue was available, the authors found a heterozygous activating mutation, either V599E or K600E, in cancerous tissue from each of 2 patients.

Conclusion: From these observations, the authors conclude that goiters resulting from thyroglobulin mutations are associated with thyroid cancer.

\section{Metastatic follicular thyroid carcinoma arising from congenital goiter as a result of a novel splice donor site mutation in the thyroglobulin gene}

Alzahrani AS, Baitei EY, Zou M, Shi Y

Department of Genetics (MBC-03), King Faisal Specialist Hospital and Research Centre, Riyadh, Saudi Arabia yufei@kfshrc.edu.sa

J Clin Endocrinol Metab 2006;91:740-746

Background: Defects in thyroglobulin ( $\mathrm{Tg}$ ) synthesis are one of the causes of thyroid dyshormonogenesis. Only a few mutations in the Tg gene have been described. The authors describe 2 siblings aged 21 and 19 years who presented with recurrent goiters for which they had undergone multiple thyroid surgeries 
since early childhood. The older sibling was diagnosed with metastatic follicular thyroid carcinoma at age 15 years.

Results: A homozygous $\mathrm{G}$ to $\mathrm{A}$ point mutation at position +1 of the splice donor site of intron 5 (g.IVS5 $+1 \mathrm{G} \rightarrow \mathrm{A}$ ) was detected in both patients, whereas a heterozygous mutation was found in their parents. RT-PCR amplification of a cDNA fragment covering exons 4-6 revealed a 191-bp fragment in the patients and 351- and 191-bp fragments in the parents. Sequence analysis of these two fragments confirmed deletion of exon 5 in the 191-bp fragment.

Conclusions: Aberrant splicing occurred as a result of the g.IVS5+1G $\rightarrow$ A mutation, which caused fusion of exons 4 and 6, resulting in the frame shift at codon position 141 and a premature stop codon at position 147 (FS141 $\rightarrow 147 \mathrm{X}$ ). The authors suggested that the malignant transformation is likely a result of prolonged TSH stimulation.

Several years ago, metastatic thyroid carcinoma was reported in an infant with congenital goiter due to mutation in the thyroperoxidase gene [J Clin Endocrinol Metab 1998;83:4162-4166]. While this former case might have been an anecdotal finding, the two independent new papers lead to some concern that cancer risk is more than a case report in patients with $\mathrm{CH}$ and dyshormonogenesis, which might have been overseen so far. Taken together, the authors report about 13 patients with $\mathrm{Tg}$ mutations who underwent surgery due to large goiters, of whom 8 were found to have follicular cancer, some of them metastatic. Both groups argue that increased TSH might be responsible for malignant tumor growth. We should be aware about a possibly increasing risk for secondary thyroid cancer in $\mathrm{CH}$ patients since they nowadays have a normal long-life expectancy. Dyshormonogenesis is not rare, and it should be possible to find out whether this is a real risk before we subject all our patients to periodic ultrasonography.

\section{Familial thyroid cancer: from prediction to prevention}

\section{Prophylactic thyroidectomy in multiple endocrine neoplasia type 2A}

Skinner MA, Moley JA, Dilley WG, Owzar K, Debenedetti MK, Wells SA Jr

Department of Surgery, Duke University School of Medicine, Durham, N.C., USA

N Engl J Med 2005;353:1105-1113

Background: Medullary thyroid carcinoma is the most common cause of death in patients with multiple endocrine neoplasia (MEN) type 2A (MEN-2A) or type 2B or familial medullary thyroid carcinoma. Total thyroidectomy in asymptomatic young members of kindreds with MEN-2A who had a mutated allele of the RET protooncogene could prevent or cure medullary thyroid carcinoma. The authors studied a total of 50 patients, 19 years of age or younger, who were consecutively identified through a genetic screening program as carriers of a RET mutation characteristic of MEN-2A and who underwent total thyroidectomy. Five to 10 years after the surgery, each patient was evaluated by physical examination and by determination of plasma calcitonin levels after stimulation with provocative agents.

Results: In 44 of the 50 patients, basal and stimulated plasma calcitonin levels were at or below the limits of detection of the assay (proportion $0.88 ; 95 \%$ confidence interval $0.76-0.95$ ). Two patients had basal and stimulated plasma calcitonin levels above the normal range. Stimulated plasma calcitonin levels had increased but remained within the normal range in 4 patients. The data suggest that there was a lower incidence of persistent or recurrent disease in children who underwent total thyroidectomy before 8 years of age and in children in whom there were no metastases to cervical lymph nodes.

Conclusion: In this study, young patients identified by direct DNA analysis as carriers of a RET mutation characteristic of MEN-2A had no evidence of persistent or recurrent medullary thyroid carcinoma 5 or more years after total thyroidectomy. A longer period of evaluation will be necessary to confirm that they are cured.

These follow-up data of patients who underwent complete thyriodectomy due to a positive genetic result for a RET protooncogene mutation is a wonderful success of preventive medicine. All patients who were operated and who were free of medullary thyroid cancer at that time were free of cancer 
5 or more years later. Before the introduction of genetic testing these individuals would have developed MTC sooner or later in life, and several of them would have developed metastases without any chance of cure. Now these individuals with RET-gene mutations can hope for a normal life with only a daily $L$-thyroxine medication after complete thyroidectomy, provided that they do not manifest other features of MEN2. For the future, the exact time point of prophylactic thyroidectomy needs to be determined and in contrast to the authors' protocol it seems that this decision should be guided by the genotype; it has been shown that specific mutations tend to result in earlier tumors than others.

\section{Familial risks for non-medullary thyroid cancer}

Hemminki K, Eng C, Chen B

Division of Molecular Genetic Epidemiology, German Cancer Research Center, Heidelberg, Germany

K.Hemminki@dkfz.de

J Clin Endocrinol Metab 2005;90:5747-5753

Background: Some data suggest that non-medullary thyroid cell cancers occur more frequently in families with affected individuals.

Methods: The authors intended to define the familial risks for histopathology-specific non-medullary thyroid cancers through parental and sibling probands. They examined the nationwide Swedish FamilyCancer Database on 10.5 million individuals, containing families with parents and offspring and cancer data from the Swedish Cancer Registry from years 1958 to 2002, including 3,292 patients with thyroid adenocarcinoma.

Results: The Systematized Nomenclature of Medicine histology was available from 1993 onwards, with 1,449 papillary, 288 follicular, 148 anaplastic, and 68 Hurthle cell tumors. The familial risk for papillary carcinoma was 3.21 and 6.24 when a parent and a sibling, respectively, were diagnosed with thyroid cancers. There was an apparent gender preference, particularly among sisters, whose risk was 11.19. The risks were highest for early-onset cancers. Thyroid adenocarcinoma was shown to be associated with melanoma and connective tissue tumors, and probably also with neurinomas (schwannomas).

Conclusion: The present findings were based on a limited number of cases, but they display a complex and heterogeneous pattern of familial non-medullary thyroid cancer. The high risk for papillary carcinoma among women requires clinical attention, although the absolute risks for this rare cancer are still low.

This paper demonstrates the power of epidemiology when cohorts of 10 million individuals are available. The main message is that relatives and especially children of patients with thyroid cancer are at statistically significant risk for the same tumor, but that the absolute risk is relatively low. The other message is of female predominance, parallels to female predominance in congenital hypothyroidism and in thyroid autoimmune disease. The intriguing question remains: Why?

\section{One hundred years later and still new concepts: autoimmune thyroid disease}

\section{A genome-wide screen in 1,119 relative pairs with autoimmune thyroid disease}

Taylor JC, Gough SC, Hunt PJ, Brix TH, Chatterjee K, Connell JM, Franklyn JA, Hegedus L, Robinson BG, Wiersinga WM, Wass JA, Zabaneh D, Mackay I, Weetman AP

Oxagen Ltd and Oxford Genetics Knowledge Park, Wellcome Trust Center for Human Genetics,

Headington, Oxford, UK

jenny.taylor@well.ox.ac.uk

J Clin Endocrinol Metab 2006;91:646-653

Background: Autoimmune thyroid diseases (AITD), comprising Graves' disease and autoimmune hypothyroidism, are characterized by loss of immunological self-tolerance to thyroid antigens. These are complex diseases arising from a combination of genetic and environmental factors. An understanding 
of the genetic susceptibility factors for AITD could help to target treatments more effectively and identify people at risk for these conditions. The objective of this study was to identify regions of genetic linkage to AITD that could potentially harbor genetic susceptibility factors for these conditions.

Results: Three regions of only suggestive but not significant linkage were obtained on chromosomes 18p11 (maximum LOD score, 2.5), 2q36 (maximum LOD score, 2.2), and 11p15 (maximum LOD score, 2.0). No linkage to human leukocyte antigen was found.

Conclusion: The absence of significant evidence of linkage at any one locus in such a large dataset argues that genetic susceptibility to AITD reflects a number of loci, each with a modest effect. Linkage analysis may be limited in defining such loci, and large-scale association studies may prove to be more useful in identifying genetic susceptibility factors for AITD.

\title{
High frequency of skewed X-chromosome inactivation in females with autoimmune thyroid disease: a possible explanation for the female predisposition to thyroid autoimmunity
}

\author{
Brix TH, Knudsen GP, Kristiansen M, Kyvik KO, Orstavik KH, Hegedus L \\ Department of Endocrinology, Odense University Hospital, Odense, Denmark \\ thomas.brix@ouh.fyns-amt.dk \\ J Clin Endocrinol Metab 2005;90:5949-5953
}

Background: Theoretically, X-chromosome inactivation (XCI) and resultant tissue chimerism could offer an explanation for the female predisposition to AITD. The aim of this study was to examine whether skewed XCI is associated with AITD. The authors first conducted a classical case-control study of twin individuals with and without AITD, and then a case-control study of twin pairs discordant for AITD, including 32 female twins with AITD and a control group of 96 healthy female twin individuals.

Results: The frequency of skewed XCI in female twins with AITD, GD, and HT was 34, 37, and 31\%, respectively, which was higher than the prevalence in the corresponding control populations, $11 \%$ $(\mathrm{p}=0.003), 14 \%(\mathrm{p}=0.045)$, and $8 \%(\mathrm{p}=0.057)$, respectively. Similar results were found in twin pairs discordant for AITD. Overall, skewed XCI was associated with an increased risk of developing AITD, with an odds ratio of 9.0 (95\% confidence interval 1.64-49.4) $(\mathrm{p}=0.022)$.

Conclusion: An odds ratio of 9.0 for AITD in skewed XCI is the highest risk factor for AITD described so far. These observations suggest a possible role of XCI in the etiology of AITD and may in part explain the female preponderance of AITD.

\begin{abstract}
More than 100 years after the first clinical descriptions of autoimmune thyroid diseases (AITD), we do not have a clear picture about the molecular mechanisms leading to this very common autoimmune disease. In that respect, AITD is one of several complex diseases that may depend on polymorphism in several genes. Like thyroid cancer, AITD occurs much more frequently in females, and female puberty seems to be part of the trigger leading to clinical manifestations. In addition, AITD runs in families suggesting a strong genetic influence. Based on these two epidemiological facts, one might expect that some factor in females triggers a genetic predisposition, which remains unknown after the large-scale study of Taylor et al. The consortium of several centers brought together 1,119 families with AITD, but failed to identify a single significant locus linked to AITD. The second paper opens an interesting view on the female predominance in AITD, speculating about an unequal X-chromosome inactivation. Skewed X-chromosome inactivation occurs in different tissues, and in the thyroid other alleles are expressed than in the thymus. This might confuse the immune system to not exclude all self-reactive T cells, thus leading to AITD. Indeed, the authors found a more frequent skewed inactivation pattern in female AITD patients. Many studies are needed in the future to see if this really plays a role in the pathogenesis of AITD, but it is a very interesting start.
\end{abstract}

